Editorial

\title{
Thirsty Kidneys: Case Report
}

\section{G. Sangeetha ${ }^{1}$, Ramasamy S. ${ }^{2}$, Rajan M. ${ }^{3}$, James.$^{4}$}

${ }^{1}$ Dr. G. Sangeetha, Fellowship in Pediatric Nephrology, Assistant Professor, ${ }^{2}$ Dr. Sunitha Ramasamy, $2^{\text {nd }}$ year Post Graduate, ${ }^{3}$ Dr. Mahalakshmi Rajan, Assistant Professor, ${ }^{4}$ Dr. Saji James, Professor; all authors are attached with Department of Pediatrics Medicine, Sri Ramachandra Medical College and Research Institute, Porur, Chennai.

Corresponding Author: Dr. G. Sangeetha, Fellowship in Pediatric Nephrology, Assistant Professor, Department of Pediatrics and Division of Pediatric Nephrology, Sri Ramachandra Medical College and Research Institute, Porur, Chennai. Email: sangeethaperungo@gmail.com

\begin{abstract}
Contrast induced nephropathy is a reversible form of acute kidney injury occurs following administration of radiocontrast agents. It is associated with serious adverse short and long-term outcomes. The risk is negligible in children with normal renal function but increased in children with underlying kidney disease, dehydration and on nephrotoxic drugs. Contrast induced nephropathy is defined as increase in serum creatinine by $>0.5 \mathrm{mg} / \mathrm{dL}$ or $25 \%$ increase from baseline within 48 to 72 hours of contrast administration. It is one of the commonest cause of hospital acquired acute kidney injury. We present a case of 10 years old boy with normal kidney function who developed acute kidney injury following intravenous contrast administration.
\end{abstract}

Keywords: Acute kidney injury, Contrast nephropathy, Hemodialysis

\section{Introduction}

Acute kidney injury (AKI) is one of the independent risk factor for mortality and morbidity in critically ill hospitalized children [1,2]. Nephrotoxic medications are the frequently attributed cause of AKI in these children. Nonsteroidal anti-inflammatory drugs (NSAIDs), angiotensin-converting enzyme inhibitors, antibiotics and radio contrast agents are the frequently encountered drugs as a cause for AKI in this group. Contrast induced AKI (CI AKI) is one of the leading causes of hospital acquired AKI. It is also known to increase the duration of hospital stay significantly.

\section{Case History}

A previously well 10 years old boy presented with fever, abdominal pain and vomiting of 3 days duration. History of blunt abdominal injury a month ago while he was riding a bicycle. His urine output was good. He was evaluated with ultrasound abdomen elsewhere revealed splenic cyst with hemorrhage, hence started on antibiotics (Inj. Amikacin) and referred to our centre for further management. On arrival he was afebrile with the heart rate of $100 / \mathrm{min}$, respiratory rate of $24 / \mathrm{min}$, BP of $100 / 70 \mathrm{mmHg}$. He had tenderness over epigastrium, umbilical and left iliac region. Other system examinations were not contributory.

His investigations showed hemoglobin of $10.8 \mathrm{~g} / \mathrm{dL}$, total white blood cell counts of $20,300 /$ cumm with $72 \%$ polymorphic predominance, sterile blood culture, normal blood urea nitrogen $(6 \mathrm{mg} / \mathrm{dL})$, serum creatinine $(0.4 \mathrm{mg} / \mathrm{dL})$, serum electrolytes and urine routine. Serum amylase was mildly elevated, $125 \mathrm{u} / \mathrm{L}$ and serum lipase was normal. Contrast enhanced computed tomography of abdomen showed laceration at the tail of pancreas with well-defined cyst in peri pancreatic region anterior to tail of pancreas suggestive of pancreatic pseudo cyst. There was no pathology in other organs. He underwent laparoscopic cysto gastrostomy after approximately 40 hours of contrast study. His urine output was nil intra as well as post operatively. He did not respond to fluid challenge. Other wise he was hemodynamically stable. Repeat blood urea nitrogen was $36 \mathrm{mg} / \mathrm{dL}$, serum creatinine was $4.1 \mathrm{mg} / \mathrm{dL}$ and electrolytes were within normal limits.

Manuscript received: $4^{\text {th }}$ February 2018

Reviewed: $12^{\text {th }}$ February 2018

Author Corrected: $18^{\text {th }}$ February 2018

Accepted for Publication: $22^{\text {nd }}$ February 2018 
In view of anuric AKI with significant elevated creatinine, hemodialysis (HD) was initiated. Using 10 Fr double lumen catheter, right femoral vein was accessed, HD was done with F4 dialyser, with the blood flow of $150 \mathrm{ml} / \mathrm{min}$, dialysate flow of $300 \mathrm{ml} / \mathrm{min}$, concurrent dialysis for the initial session and subsequent countercurrent dialysis with a total of $4 \mathrm{HD}$ sessions. Repeat CT abdomen on 3rd post-operative day showed bilateral renomegaly and mild dilatation of proximal ureter with distal tapering on both sides [Figure 1\&2]. Hence viscous contrast material blocking both side ureter causing oliguric AKI was considered as the cause. He started making urine on 4th post-operative day and gradually urine output improved to $2 \mathrm{ml} / \mathrm{kg} /$ day. He was discharged on $12^{\text {th }}$ postoperative day with the creatinine of $0.3 \mathrm{mg} / \mathrm{dL}$.

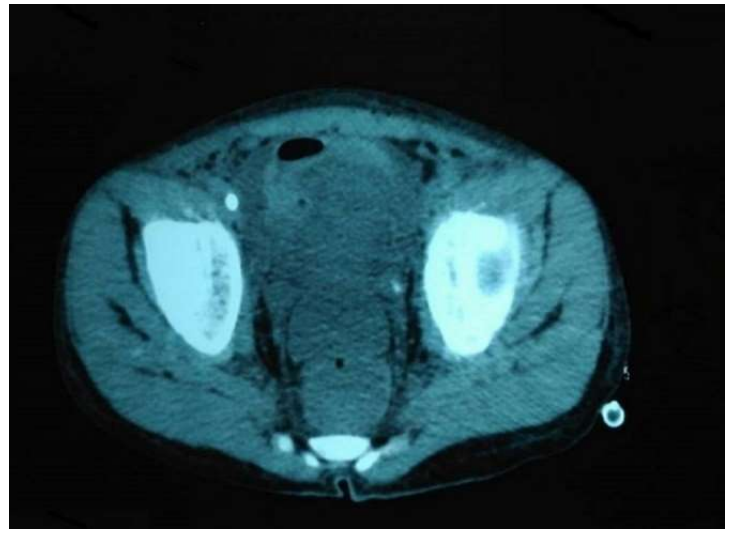

Figure-1: Bilateral enlarged kidneys

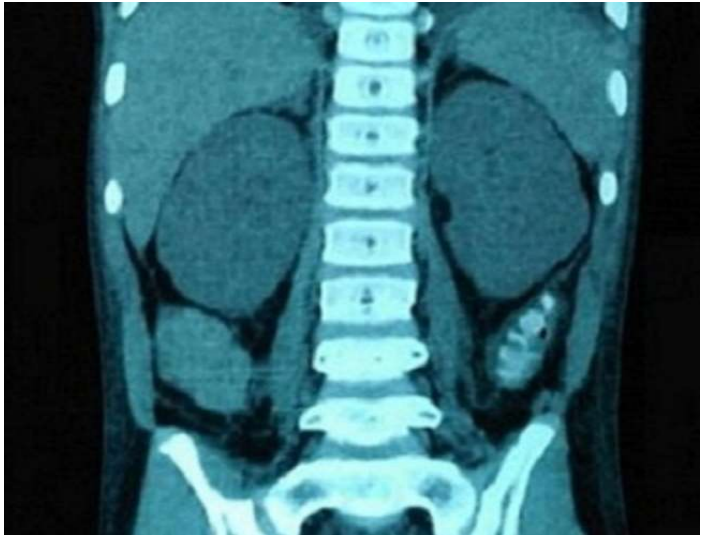

Figure-2: Bilateral enlarged kidneys

\section{Discussion}

The prevalence of AKI in children who need intensive care varies between $5 \%$ to over $80 \%$ depending upon the underlying causes and co morbidities [3,4]. More than $80 \%$ of hospitalized children are exposed to at least one nephro toxic medication during their hospital stay. Contrast induced acute kidney injury is a reversible form of AKI. It is defined as rise in serum creatinine of more than $0.5 \mathrm{mg} / \mathrm{dL}$ or a $25 \%$ increase from baseline value assessed at 48 hours after a radiological procedure [5]. Other possible causes of AKI should be ruled out before making the diagnosis of CIAKI. Our patient also diagnosed as CI AKI after ruling out other possible causes of AKI like sepsis, dehydration. He received 2 doses of aminoglycoside prior to admission which might be the additive factor for the development of AKI. The incidence of CI AKI in adult is $18 \%$ and is one of the major cause of hospital acquired kidney injury [5]. A study conducted by Ajami G et al showed the incidence of CI AKI as $18.75 \%$ in 80 children who had undergone cardiac angiography [6].

Serum creatinine usually peaks at 3 to 5 days after contrast administration and returns to baseline or near baseline within a couple of weeks. Common presentation of contrast induced AKI is non-oliguric renal failure and does not need renal replacement therapy. Since this boy presented with anuric AKI, he had been initiated on hemodialysis. Risk factors can be divided as patient related and procedure related. Pre-existing renal insufficiency, volume depletion like diarrhoea, vomiting, poor oral intake and long term nephrotoxic drug intake are patient related risk factors whereas procedure related factors include dose of contrast, osmolality as well as viscosity and route of administration. Iso/low osmololnon-ionic second and third generation contrast agents are preferred in view of lesser adverse effects [7]. Our child received second generation contrast agent for his study. The pathogenesis of contrast induced AKI is not completely understood.

The accepted couple of theories are focusing on renal tubular injury. Intravenous contrast ingestion causes renal vasoconstriction results in medullary hypoxia which is probably mediated by alterations in nitric oxide, endothelin, and adenosine. Second possible theory is that acute tubular injury is a direct cytotoxic effect of the contrast agents [8]. As the serum creatinine rises only after 48 hours of renal injury, other sensitive early biomarkers such as plasma neutrophil gelatinaseassociated lipocalin (NGAL), plasma cystatin C, urinary interleukin 18 and urinary kidney injury molecule-1 (KIM-1) may be helpful to find out the injury earlier [9].

Non-pharmacological measures like maintaining good hydration and monitoring urine output are the mainstay of prevention of contrast induced AKI. Normal saline is 
Editorial

preferred rather than other intravenous fluids as it maintains good intravascular volume and prevents renin angiotensin axis activation [10,11]. It is ideal to maintain urine output of $>1$ to $1.5 \mathrm{ml} / \mathrm{kg} /$ hour 3 to 12 hours pre-contrast period and for 6 to 12 hours following contrast administration[5]. Always use lower possible dose of contrast medium. Avoid repetitive studies closely spaced within 48 to $72 \mathrm{hrs}$.

Pharmaco-logical prevention strategies are discontinuation of nephrotoxic drugs at least 24 to 48 hours prior to contrast administration. Sodium bicarbonate and antioxidant $\mathrm{N}$-acetylcysteine are being tried though there is no clear evidence-based guidelines [12]. Always repeat the renal function test at 24 and $48-72 \mathrm{hrs}$ after the procedure to find out any alteration in renal function and to decide about management.

Funding: Nil, Conflict of interest: None initiated,

Perission from IRB: Yes

\section{References}

1. Plotz FB, Bouma AB, Van Wijk JA, Kneyber MC, Bokenkamp A. Pediatric acute kidney injury in the ICU: An independent evaluation of pRIFLE criteria. Intensive Care Med. 2008; 34 (9): 1713-1717.doi: 10. 1007 /s 00134-008-1176-7.

2. Morgan CJ, Zappitelli M, Robertson CM, Alton GY, Sauve RS, Joffe AR, et al. Risk factors and outcomes of acute kidney injury in neonates undergoing complex cardiac surgery. J Pediatr. 2013;162(1): 120-127. doi: 10. 1016/j.jpeds.2012.06.054.

3. Slater MB, Anand V, Uleryk EM, Parshuram CS. A systematic reviewof RIFLE criteriain children, and its application and association with measures of mortality and morbidity. Kidney Int.2012; 81:791-798.doi:10. 1038/ki.2011.466.

4. John R. Prowle. Acute kidney injury: an intensivist's perspective. PediatrNephrol. 2014; 29(1):13-21.doi: 10. 1007/s00467-013-2411-1.
5. Contrast-induced AKI Kidney International Supplements. KDIGO Clinical Practice Guideline for Acute Kidney Injury. 2012; 2:69-88; doi:10.1038/ kisup. 2011.34.

6. Ajami G, Derakhshan A, Amoozgar H, Mohamadi $\mathrm{M}$, Borzouee $\mathrm{M}$, Basiratnia $\mathrm{M}$, et al. Risk of Nephropathy After Consumption of NonionicContrast Media by Children Undergoing Cardiac Angiography: A Prospective Study. PediatrCardiol 2010;31: 668-673. doi:10.1007/s00246-010-9680-2.

7. Heyman SN, Rosenberger C, Rosen S. Regional alterations in renal haemodynamics and oxygenation: a role in contrast medium-induced nephropathy. Nephrol Dial Transplant 2005; 20 :1-i6-i11. doi:10.1093/ ndt/gfh1069.

8. Detrenis S, Meschi M, Musini S, Savazzi G. Lights and shadows on the pathogenesis of contrast-induced nephropathy: state of the art. Nephrol Dial Transplant. 2005; 20:1542- 1550. doi:10.1093/ndt/gfh868.

9. Haase M, Devarajan P, Haase-Fielitz A, Bellomo R, Cruz DN, Wagener G, et al. The outcome of neutrophil gelatinase-associated lipocalin-positive subclinical acute kidney injury: A multicenter pooled analysis of prospective studies. J Am Coll Cardiol.2011; 57(17): 1752-176.doi: 10.1016/j.jacc. 2010.11.051.

10. Mueller C. Prevention of contrast-induced nephropathy with volume supplementation. Kidney Int Suppl.2006;69:S16-S19.doi.org/10.1038/sj.ki. 5000369.

11. Pannu N, Wiebe N, Tonelli M. Prophylaxis strategies for contrast-induced nephropathy. JAMA. 2006 Jun 21. 295; 23:2765-2779. doi: 10.1001/jama. 295. 23.2765.

12. Steven Fishbane. N-Acetylcysteine in the Prevention of Contrast-Induced Nephropathy. Clin J Am Soc Nep hrol. 2008; 3: 281-287. doi:0. 2215/ CJN. 02590607

\section{How to cite this article?}

G. Sangeetha, Ramasamy S, Rajan M, James S. Thirsty Kidneys: Case Report. Int J Pediatr Res. 2018;5(2):100-102. doi:10.17511/ijpr.2018.i02.10. 